\title{
Voltage Dependence of NMDA-Activated Macroscopic Conductances Predicted by Single-Channel Kinetics
}

\author{
Craig E. Jahr ${ }^{1}$ and Charles F. Stevens ${ }^{2}$ \\ 'The Vollum Institute, Oregon Health Sciences University, Portland, Oregon 97201, and ${ }^{2}$ The Salk Institute, San Diego, \\ California 92138
}

\begin{abstract}
The conductance activated in many mammalian CNS neurons by the glutamate analog NMDA is inhibited at hyperpolarized potentials by extracellular magnesium. Whole-cell recordings from hippocampal neurons in culture were used to determine the voltage dependence of the NMDA conductance in the presence of extracellular magnesium concentrations from $1 \mu \mathrm{M}$ to $10 \mathrm{~mm}$. The conductance-voltage data are well fitted by a gating function derived from rate constants determined in an earlier study of the kinetic behavior of single channels activated by NMDA. The results are consistent with the assumption that magnesium inhibits current through the NMDA-activated channel by directly blocking the ion pore. In addition, another voltage-dependent blocking or flicker-producing mechanism has to be invoked to account for the behavior of the conductance at both the single-channel and whole-cell level, especially at low concentrations of extracellular magnesium.
\end{abstract}

In the presence of extracellular magnesium, single NMDA-receptor channels exhibit bursts of brief openings separated by short intervals during which no current flows (Nowak et al., 1984) that we call interruptions. We have shown in a previous study (Jahr and Stevens, 1990) that a 3- or 4-state model with closed and open states and 1 or 2 "blocked" states in communication with both closed and open states can accurately describe this single-channel behavior. Our previous work, however, left 2 important questions unanswered. The first deals with the physical mechanism underlying the kinetic scheme that accounts for single-channel behavior, and the second addresses the implications of the described single-channel behavior for macroscopic currents.

According to the 3-state model, a channel that starts in an open state $(\mathrm{O})$ can make transitions into 2 distinct nonconducting states called "closed" (C) and "blocked" (B):

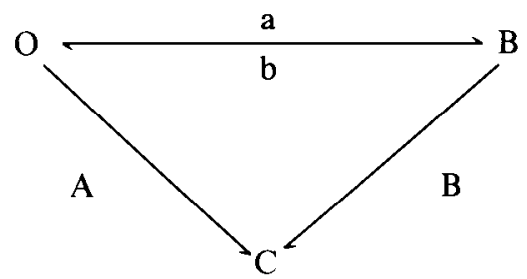

Received Feb. 26, 1990; revised Apr. 23, 1990; accepted May 1, 1990.

This work was supported by NIH Grants NS21419 (to C.E.J.) and NS12961 (to C.F.S.), The McKnight Endowment Fund for Neuroscience (to C.E.J.), and the Howard Hughes Medical Institute (to C.F.S.).

Correspondence should be addressed to Craig E. Jahr, Vollum Institue L474, Oregon Health Sciences University, 3181 Southwest Sam Jackson Park Road, Portland, OR 97201.

Copyright (c) 1990 Society for Neuroscience $0270-6474 / 90 / 093178-05 \$ 03.00 / 0$
It is the repeated transitions between $\mathrm{O}$ and $\mathrm{B}$, finally terminated by the entry into the long-lived state $C$, that constitutes a burst. The rates of transition between $\mathrm{O}$ and $\mathrm{B}$ depend exponentially on voltage, and the $\mathrm{O}-\mathrm{B}$ transition rate increases approximately linearly with the concentration of magnesium in the extracellular medium. The rates from both $\mathrm{O}$ and $\mathrm{B}$ into state $\mathrm{C}$ are independent of voltage and magnesium concentration.

The physical question at issue is the origin of the dependence on magnesium concentration and voltage of the rate constants between the $\mathrm{O}$ and $\mathrm{B}$ states. The rate at which interruptions of the $\mathrm{O}$ state occur, that is, brief transitions from $\mathrm{O}$ to $\mathrm{B}$, are found experimentally to depend on the extracellular magnesium concentration, and this could occur in at least 2 (limiting) ways: the $\mathrm{O}-\mathrm{B}$ transition could be a voltage-dependent conformational change that is enabled by the voltage-independent binding of magnesium to some site, or the $\mathrm{O}-\mathrm{B}$ transition could derive its voltage dependence from the movement of magnesium to a blocking site located partway through the voltage difference between the outside and inside of the membrane. Both of these physical mechanisms would give rise to the same scheme shown above, but the molecular interpretation of interruptions would be quite different in the 2 cases. In this paper, we present information that favors the blocking mechanism.

Macroscopic currents activated by NMDA have a markedly nonlinear current-voltage relationship (MacDonald and Wojtowicz, 1982), the shape of which is dependent on extracellular magnesium concentration (Mayer et al., 1984; Novak et al., 1984). The nonlinearity has been attributed to magnesium blocking the open channel (Mayer et al., 1984; Nowak et al., 1984). To determine if this interpretation is correct and to provide a quantitative description of the voltage-dependent gating properties of NMDA-receptor channels, we use the 4-state model described in an earlier paper (Jahr and Stevens, 1990) to derive a gating function that relates the fraction of time activated channels conduct current to the membrane potential; this model accurately predicts macroscopic currents over a range of magnesium concentrations. The gating function derived in this way has no free parameters, because all constants have been determined in the previous single-channel analysis. We can, therefore, test the adequacy of this formulation over a much wider range of magnesium concentrations than is possible with singlechannel recording and can obtain information, as described below, relcvant to the physical interpretation of the formal kinetic scheme.

\section{Materials and Methods}

The methods are the same as those used in the earlier paper (Jahr and Stevens, 1990). Test solutions were applied to whole cells either with pressure ejection from pipettes with tip diameters of about $4-8 \mu \mathrm{m}$ or 
flow pipes with internal diameters of $300-400 \mu \mathrm{m}$ (Johnson and Ascher, 1987). Drug applications with pressure pipettes positioned $10-20 \mu \mathrm{m}$ from the soma lasted $2 \mathrm{sec}$. Flow-tube applications lasted $2-10 \mathrm{sec}$ and had onsets fast cnough to obscrve a desensitizing component and a steady-state component of the response. Amplitude measurements were taken at steady state. Results from both application techniques were indistinguishable. NMDA and glycine concentrations were constant at $30 \mu \mathrm{M}$ and $1 \mu \mathrm{M}$, respectively, in all experiments.

\section{Results}

In the earlier single-channel analysis (Jahr and Stevens, 1990), we could not use magnesium concentrations greater than about $0.2 \mathrm{~mm}$ because the interruption rate became so high that we were unable to resolve individual transitions. The key to testing whether we have developed a satisfactory description for singlechannel behavior, then, is to derive consequences of our theory that can be examined within and above the physiological range of magnesium concentrations. We shall derive a gating function for macroscopic currents based on the rate constants derived from single-channel experiments (Jahr and Stevens, 1990) and show that we can account for the NMDA-receptor-channel voltage dependence without free parameters over a wide range of magnesium concentrations. We start by deriving an expression for the gating function, then compare its predictions with experimental observations.

The gating function. Single NMDA-receptor-channel open behavior is accurately described by the 4-state model:

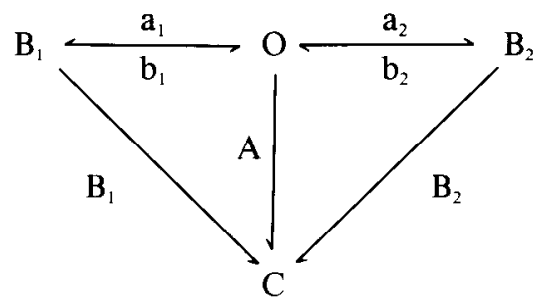

where the transition rates are given in Table 1. Because opening rates, that is, the $\mathrm{C}-\mathrm{O}$ transition, are tcchnically difficult to study, we must make the assumption that these rates are not significantly influenced by voltage; this means that, for a fixed agonist concentration, the entire gating function depends only on the fraction of time channels spend in the $O$ rather than $B$ states.

The gating function $\mathrm{g}(V)$, where $V$ is membrane potential, is the conductance contributed per channel burst relative to the amount of conductance that a burst would give at very positive voltages where very few interruptions occur. The average number of interruptions per burst is defined to be $n$, so that the number of intervals in $\mathrm{O}$ (the conducting state) is $(n+1)$ and the mean dwell time in the $\mathrm{O}$ state each time it is entered is denoted $t_{\mathrm{o}}$. The mean total time a channel spends in the $\mathrm{O}$ state during each burst is therefore $(n+1) t_{\mathrm{O}}$. The gating function is thus:

$$
\mathrm{g}(V)=(n+1) t_{\mathrm{o}} /(\text { open time per burst for large } V) .
$$

Note that both $n$ and $t_{\circ}$ depend on $V$; their explicit dependence will be calculated later.

As $V$ becomes large and positive, the rate constants $a_{1}$ and $a_{2}$, for entering the blocked states, approach 0 by virtue of their voltage dependence. Because interruptions are the result of entering states $B_{1}$ and $B_{2}$, the average number of interruptions per burst $(n)$ approaches 0 as $V$ becomes large, so that the total open

\section{Table 1. Rate constants for 4-state model}

\begin{tabular}{ll}
$\begin{array}{l}\text { Rate } \\
\text { constant }\end{array}$ & Value \\
\hline $\mathrm{a}_{1}$ & $\exp (-0.016 \mathrm{~V}-2.91) \mathrm{msec}^{-1}$ \\
$\mathrm{a}_{2}$ & $C \exp (-0.045 \mathrm{~V}-6.97) \mu \mathrm{M}^{-1} \mathrm{msec}^{-1}$ \\
$\mathrm{~b}_{1}$ & $\exp (0.009 \mathrm{~V}+1.22) \mathrm{msec}^{-1}$ \\
$\mathrm{~b}_{2}$ & $\exp (0.017 \mathrm{~V}+0.96) \mathrm{msec}^{1}$ \\
$\mathrm{~A}$ & $\exp (-2.847) \mathrm{msec}^{-1}$ \\
$\mathrm{~B}_{1}$ & $\exp (-0.693) \mathrm{msec}^{-1}$ \\
$\mathrm{~B}_{2}$ & $\exp (-3.101) \mathrm{msec}^{-1}$
\end{tabular}

Rate constants for the 4-state model were derived from single-channel analysis (see Jahr and Stevens, 1990). $C$ is the magnesium concentration in $\mu \mathrm{M} ; V$ is the voltage in $\mathrm{mV}$.

time per burst approaches $t_{\mathrm{O}}$. The mean open time is given by

$$
t_{\mathrm{O}}=1 /\left(\mathrm{a}_{1}+\mathrm{a}_{2}+\mathrm{A}\right) \text {, }
$$

that is, the reciprocal of the rates for leaving the $O$ state. As $V$ becomes large, $\mathrm{a}_{1}$ and $\mathrm{a}_{2}$ approach 0 and $t_{\mathrm{O}}$ approaches $1 / \mathrm{A}$ (note that $\mathrm{A}$ is voltage independent). The open time per burst for a large $V$ is $1 / \mathrm{A}$ and the gating function is then

$$
\mathrm{g}(V)=\mathrm{A}(n+1) t_{\mathrm{O}}
$$

To find $\mathbf{g}(V)$ in terms of the rate constants, we need an expression for $n$; Equation (1) already gives $t_{\mathrm{o}}$ in terms of closing rates, and $A$ is simply a voltage-independent scale factor. Using the expression for $n$ presented in the carlier paper (Jahr and Stevens, 1990), we find that $(n+1)$ is given by

$$
(n+1)=1 /\left(1-\mathrm{FG} t_{i} t_{\mathrm{O}}\right)
$$

where $F=\left(a_{1}+a_{2}\right), G=\left(b_{1} a_{1}+b_{2} a_{2}\right) /\left(a_{1}+a_{2}\right)$, and $t_{i}=\left(a_{1}+\right.$ $\left.a_{2}\right) /\left[a_{1}\left(b_{1}+B_{1}\right)+a_{2}\left(b_{2}+B_{2}\right)\right]$. When $g(V)$ is expressed in terms of rate constants by using Equations (1) and (3), we obtain, after some algebraic manipulations,

$$
\begin{aligned}
g(V)=1 /\left\{1+\left(a_{1}+a_{2}\right)\left(a_{1} B_{1}+a_{2} B_{2}\right) /\right. & {\left[A_{1}\left(b_{1}+B_{1}\right)\right.} \\
& \left.\left.+A_{2}\left(b_{2}+B_{2}\right)\right]\right\} .
\end{aligned}
$$

From our single-channel analysis, we know that $b_{1}$ and $b_{2}$ are much larger than $B_{1}$ and $B_{2}$, so Equation (4a) is, to a good approximation:

$$
g(V)=1 /\left[1+\left(a_{1}+a_{2}\right)\left(a_{1} B_{1}+a_{2} B_{2}\right) / A\left(a_{1} b_{1}+a_{2} b_{2}\right)\right] .
$$

When the magnesium concentration exceeds a few hundred micromolar, $a_{2}$ (which increases linearly with magnesium concentration) becomes much greater than $a_{1}$ (which is independent of magnesium concentration), so $g(V)$ should be well approximated in the physiological range of magnesium concentrations by

$$
\mathrm{g}(V)=1 /\left(1+\mathrm{B}_{2} \mathrm{a}_{2} / \mathrm{Ab}_{2}\right) \text {. }
$$

In this expression, only $a_{2}$ and $b_{2}$ are, according to our earlier experiments, voltage dependent. When values for rate constants determined from single-channel studies (Table 1) are inserted into this equation, we find, for physiological magnesium concentrations, that

$$
\mathrm{g}(V)=1 /[1+\exp (-0.062 V)(C / 3.57)]
$$

where $V$ is membrane potential in $\mathrm{mV}$, and $C$ is extracellular magnesium concentration in $\mathrm{mm}$.

Experimental observations. Figure 1 illustrates representative 
Figure 1. Current-voltage relationships of NMDA- and glycine-evoked currents in the presence of 1 and $10 \mathrm{~mm}$ external magnesium. Records on the left are voltage-clamped currents at 5 holding potentials elicited by the application of $30 \mu \mathrm{M}$ NMDA and $1 \mu \mathrm{M}$ glycine in the presence of $1 \mathrm{~mm}, 10 \mathrm{~mm}$, then back to $1 \mathrm{~mm}$ magnesium from flow pipes serially positioned in front of the neuron. On the right are complete current-voltage relationships in the 2 magnesium concentrations (diamonds, $1 \mathrm{~mm}$ magnesium; triangles, $10 \mathrm{~mm}$ magnesium). Calibration, $300 \mathrm{pA}, 2 \mathrm{sec}$.
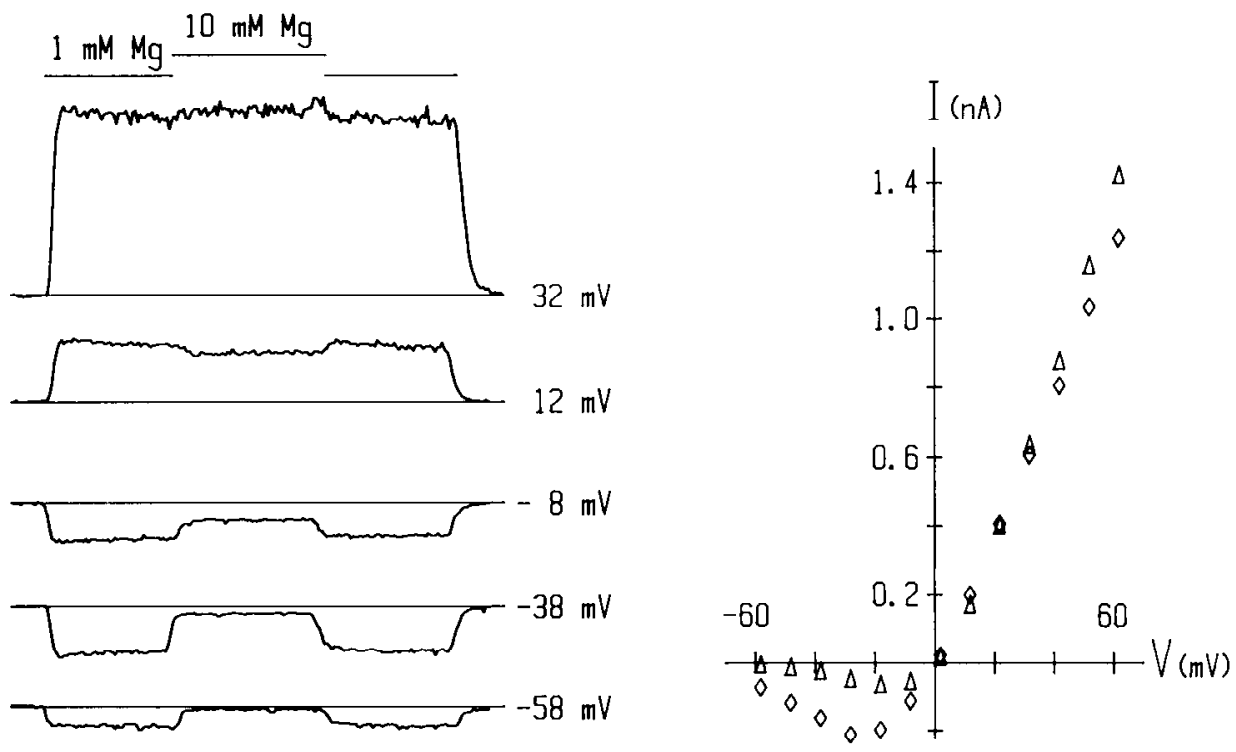

currents evoked by application of $30 \mu \mathbf{M}$ NMDA and $1 \mu \mathbf{M}$ glycine in the presence of $1 \mathrm{~mm}$ and $10 \mathrm{~mm}$ magnesium at various holding potentials. The current-voltage relation on the right plots the current amplitudes obtained in $1 \mathrm{~mm}$ and $10 \mathrm{~mm}$ magnesium from the same cell. Conductance-voltage plots were constructed (assuming a reversal potential of $0 \mathrm{mV}$ ) from these data and those obtained in the presence of $10 \mu \mathrm{M}$ and $100 \mu \mathrm{M}$ magnesium on the same cell (Fig. 2). In all concentrations of magnesium tested, blockade is completely overcome at holding potentials more positive than about $+50 \mathrm{mV}$.

The conductance versus voltage families shift to the right in an orderly fashion with increasing external magnesium, as illustrated in Figure 2; note that the gating functions become less steep with decreasing magnesium concentrations. Data from 24 current-voltage curves obtained from 12 cells were fitted with the gating function described in Equation (4a) and the rate constants in Table 1 . The voltage at which the conductance reaches half of its maximum was obtained at 11 magnesium concentrations. This quantity is plotted versus magnesium concentration in Figure 3. In addition, gating functions were calculated from single-channel data for 4 patches at 6 magnesium concentrations. These gating functions were found by plotting the total amount of time channels were open per second of observation, normalized by the maximum amount of time at positive voltages, as a function of voltage. Again, voltages at which the gating function is half-maximal were determined and plotted on Figure 3.

According to Equation (5), the gating function attains its halfmaximal value $V_{\mathrm{m}}$ when

$$
C / 3.57=\exp \left(0.062 V_{\mathrm{m}}\right)
$$

thus

$$
V_{\mathrm{m}}=\ln (C / 3.57) / 0.062
$$

or

$$
V_{\mathrm{m}}=37.1 \log (C)-20.5 \text {. }
$$

$V_{\mathrm{m}}$ then shifts $37.1 \mathrm{mV}$ for each 10 -fold change in magnesium concentration. Equation (6) is plotted as the straight line in Figure 3 and can be seen to provide an adequate characterization of the data for magnesium concentrations greater than about $300 \mu \mathbf{M}$.

It is clear from Figure 3 that the midpoints used to fit the individual conductance-voltage plots do not lie directly on the theoretical curve generated by Equation (4a). If the theoretical midpoints were used to fit the gating function to each data set, the gating curves would be shifted along the voltage axis relative to the data. This was also true with the single-channel data

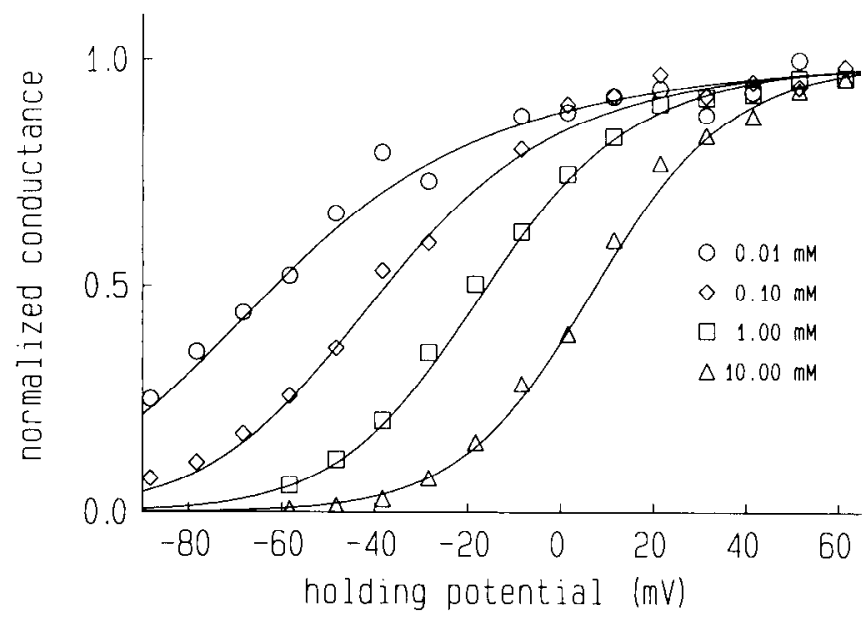

Figure 2. Conductance-voltage relationships of NMDA responses at 4 magnesium concentrations. Current amplitudes from the cell illustrated in Figure 1 were converted to conductance assuming a reversal potential of $0 \mathrm{mV}$, normalized to maximal conductance, and plotted against holding potential. The data are fitted with curves generated from the gating function derived from the 4-state model [Eq. (4a)] using the rate constants in Table 1 . Rate constant $a_{2}$ was changed from $C \exp (-0.045 V-6.97)$ to $C \exp (-0.055 V-7.1)$ (see text). 


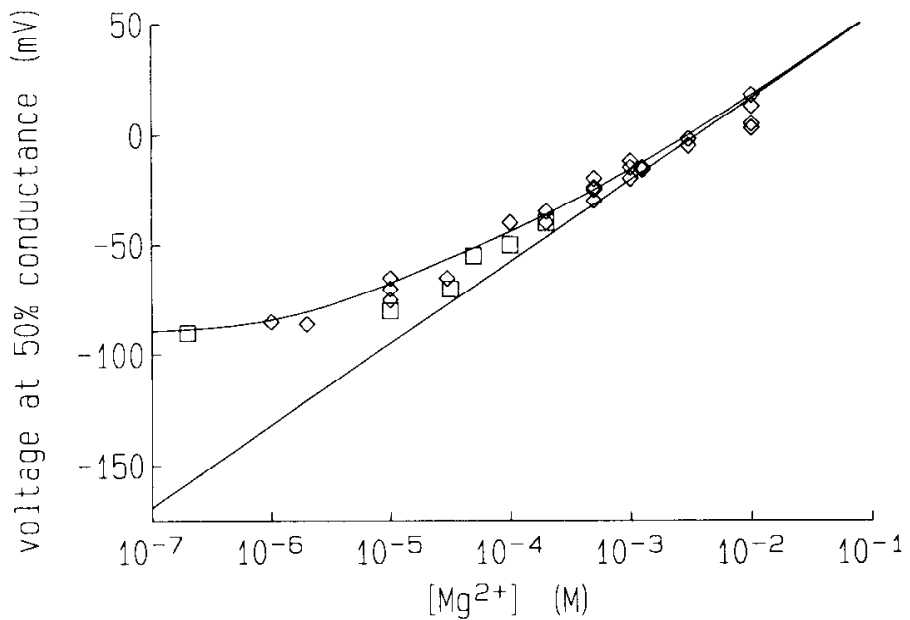

presented previously (Jahr and Stevens, 1990). Although only one set of rate constants (Table 1) were used to fit all the data, results from each individual patch were best fitted by slightly different values. Thus, the midpoints of the conductance curves and the value of the rate constant $a_{2}$ were altered to match the midpoint of the whole-cell data in Figure 2; the shape of the curve is not appreciably altered by these changes in $a_{2}$, and the midpoint is moved by a average of $3 \mathrm{mV}$. The adjustments in $\mathrm{a}_{2}$ are within the range of measurement uncertainties. The variability from one data set to the next may be accounted for by changes in temperature or by alterations in the gating properties of different populations of channels.

The data points in Figure 3 systematically deviate from the straight line at magnesium concentrations of below about 300 $\mu \mathrm{M}$. This deviation is expected because Equation (6) was derived as the limiting case for high magnesium concentrations, and lower concentration data should not fall on the line. The full gating function [Eq. (4a)], derived from the kinetic model for single-channel behavior, predicts the curved line in Figure 3. Clearly, the full gating function provides a better fit to the $V_{\mathrm{m}}$

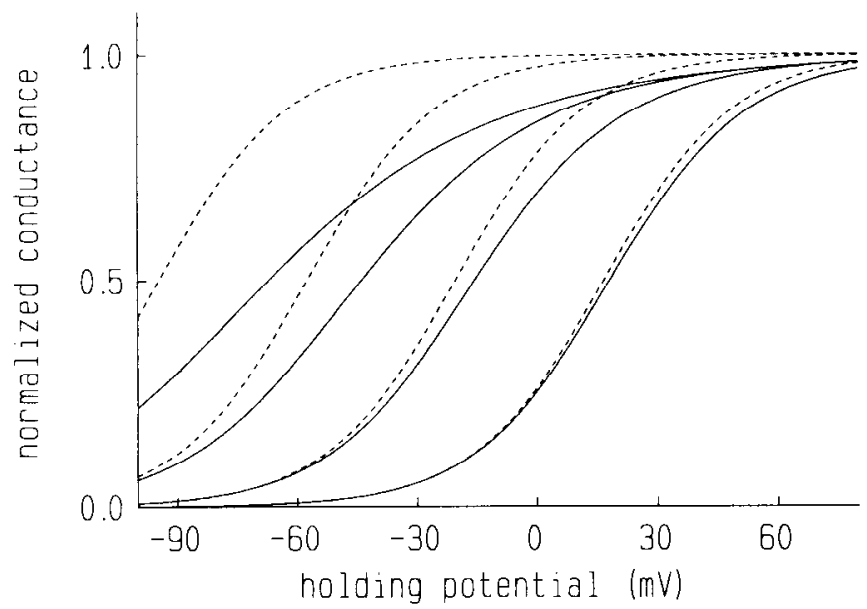

Figure 4. The full gating function [Eq. (4a)] as a function of voltage from Figure 3 (solid lines), with the limiting gating function [Eq. (5)] for high magnesium concentrations superimposed (dashed line). The magnesium concentrations for these calculations were, left to right, 0.01 , $0.1,1$, and $10 \mathrm{~mm}$. Rate constants are from Table 1 .
Figure 3. Relationship between the voltage at which the conductance is half maximal and magnesium concentration. The half-maximum voltages were obtained from plots of conductance versus voltage as in Figure 2 and plotted against magnesium concentration (diamonds, whole cell data; squares, single channel data). The data are fitted with a theoretical curve generated from Equation (4a) which assumes that a second "blocking" mechanism not dependent on magnesium concentration is predominant at low magnesium concentrations. The linear fit is derived from Equation (5), which assumes the high magnesium-concentration limit. Rate constants are from Table 1. data than does the high magnesium-concentration limit embodied in Equation (6).

From Figure 3, one might believe that the high concentration limit holds for magnesium concentrations greater than $0.3 \mathrm{~mm}$. This notion is tested in Figure 4, where predictions of Equations (4a) and (5) are superimposed. In this figure, deviations from the limiting case are pronounced below $1 \mathrm{~mm}$. Given the scatter in the $V_{\mathrm{m}}$ data (Fig. 3) and in the empirically determined gating functions (Fig. 2), the limiting equation [Eq. (5)] can probably be considered adequate for magnesium concentrations in the physiological range of about $1 \mathrm{~mm}$.

\section{Discussion}

Our results show that the voltage-dependent behavior of the NMDA-activated conductance in whole-cell recordings depends on extracellular magnesium concentration in a manner consistent with the assumption that magnesium directly blocks the ion pore. This model requires that the second-order rate constant $\mathrm{a}_{2}$ depend linearly on magnesium concentration:

$$
\mathbf{a}_{2}=C r \text {, }
$$

where $C$ is the magnesium concentration and $r$ is a magnesium concentration independent quantity. If, on the other hand, magnesium antagonized the conductance by binding to a site that allosterically altered the voltage dependence of gating, the dependence of the "blocking" rate constant on magnesium concentration $C$ would not be linear over a wide range of concentrations, but rather would saturate according to a binding equation such as

$$
\mathrm{a}_{2}=r /\left(1+\mathrm{C}_{0} / C\right) \text {; }
$$

this model would predict an approximately linear relationship over magnesium concentrations that are small compared to $\mathrm{C}_{0}$, where $\mathrm{C}_{0}$ is the dissociation constant for the binding site. In the previous single-channel study (Jahr and Stevens, 1990), we could not use magnesium concentrations high enough that one might expect obvious deviations from linearity if the second model were correct. In the present study, the NMDA conductance has been studied in the presence of magnesium in concentrations that span 4 orders of magnitude up to $10 \mathrm{~mm}$. When the rate constant describing magnesium block is separated from the nonmagnesium "blocking" process, it depends linearly on magnesium concentration, indicating either that the first model is cor- 
rect or, if the second model is correct, the dissociation constant for magnesium is greater than $10 \mathrm{~mm}$.

We conclude, then, that the kinetic model published earlier (Jahr and Stevens, 1990) provides a satisfactory description for magnesium concentrations over 4 orders of magnitude. For magnesium concentrations in the physiological range, the limiting gating function [Eq. (5)] holds, but for lower magnesium levels, the full description [Eq. (4a)] is required. Although we cannot entirely exclude the possibility that magnesium causes open-channel interruptions by enabling a voltage-dependent conformational change, our observations strongly support channel block as the physical mechanism for the inhibition of the NMDA conductance by magnesium, as originally suggested by Nowak et al. (1984) and Mayer et al. (1984).

\section{References}

Jahr CE, Stevens CF (1990) A quantitative description of NMDA receptor-channel kinetic behavior. J Neurosci 10:1830-1837.

Johnson JW, Ascher P (1987) Glycine potentiates the NMDA response in cultured mouse brain neurons. Nature 325:529-531.

MacDonald JF, Wojtowicz JM (1982) The effects of L-glutamate and its analogues upon the membrane conductance of central murine neurones in culture. Can J Physiol 60:282-296.

Mayer MI, Westbrook GI, Guthrie PB (1984) Voltage-dependent block by $\mathrm{Mg}^{2+}$ of NMDA responses in spinal cord neurones. Nature 309:261-263.

Nowak L, Bregestovski P, Ascher P, Herbet A, Prochiantz A (1984) Magnesium gates glutamate-activated channels in mouse central neurones. Nature 307:462-465. 